\title{
Semiclassical Calculations with Nuclear Model Potentials in the $\hbar$-Resummation Approach
}

\author{
J. Bartel* \\ Institut Laue-Langevin, Grenoble, France \\ and Laboratoire de Physique Théorique, Université de Bordeaux, \\ Gradignan, France \\ M. Durand \\ Institut des Sciences Nucléaires (IN2P3), Grenoble, France \\ M. Brack \\ Institut für Theoretische Physik, Universität Regensburg, \\ Federal Republic of Germany
}

Received September 26, 1983

The single-particle density $\rho(\mathbf{r})$ of a system of fermions can be calculated in a tractable way as the Laplace inverse of the Bloch density describing the system. The complex integrals involved can be solved very easily by the saddle-point method. The semiclassical nature of this approach is illustrated in the simple example of the single-particle level density of a harmonic oscillator potential. It is then applied to calculate the total energy of particles in different mean field potentials. The exact Bloch density being generally unknown, different approximate forms are used in our calculations which correspond to a partial resummation of the Wigner-Kirkwood $\hbar$-expansion. The resulting local densities reproduce the exact density distributions on the average, without quantal oscillations. They are well defined everywhere, even beyond the classical turning point, in contrast to the original Wigner-Kirkwood approach.

\section{Introduction}

It has become increasingly clear over the past few years that the semiclassical approximation constitutes a very useful approach to the nuclear many body problem. Indeed, the successes of semi-empirical mass formulae like the liquid drop or droplet model [1-5] are due to the fact that the shell correction energy is very small compared to the nuclear bulk energy. This allows for a perturbative treatment of shell effects as done for instance in the Strutinsky method $[6,7]$. To test the validity of the Strutinsky energy theorem and to calculate the shell-correction energy microscopically, it has been found essential to determine the average density matrix $\bar{\rho}$ in a selfconsistent way. This has been proved by using microscopically Strutinsky-smoothed densities in an iterative Hartree-Fock (HF) cycle [8,9].

\footnotetext{
* Present address: Institut für Theoretische Physik, Universität Regensburg, Universitätsstrasse 31, D-8400 Regensburg, FRG
}

In this paper, it will be shown how the partial $h$ resummation technique can yield average densities $\bar{\rho}$ and average energies $E(\bar{\rho})$ in a much more economical way which avoids the use of single-particle wavefunctions. Using this semiclassical method in connection with density dependent effective forces of the Skyrme type [10-12] it has been recently possible [13] to calculate in an iterative cycle self-consistent average binding energies and density distributions of spherical nuclei.

The aim of the present paper is a study of the two main ingredients used in this self-consistent semiclassical procedure: the partial $h$-resummation technique and the saddle-point method. This paper continues a series of investigations on the partial $\hbar$ resummation method [14-16]. Chapter 2 will be devoted to a short presentation of this approach. In Chap. 3, the saddle-point method will be studied in some detail and compared with the Strutinsky 
smoothing procedure. Average density distributions and energies obtained for two different model potentials will be presented in Chap. 4 and compared with the corresponding Strutinsky results.

\section{The Partial Resummation of the Wigner-Kirkwood Series}

So far there have been mainly two different, though closely related semiclassical techniques which have been used to deduce average nuclear properties, such as binding energies and density distributions, from an effective nucleon-nucleon interaction. These are the extended Thomas-Fermi method (ETF) and the partial $\hbar$-resummation technique. Both these methods use the Wigner-Kirkwood expansion [17-19] as a starting point and go beyond the Thomas-Fermi (TF) approximation.

The single particle Bloch density

$$
C_{\beta}=e^{-\beta \mathscr{H}}
$$

of a Fermion system, can be written as an expansion in Wigner space

$$
\begin{aligned}
C(\mathbf{r}, \mathbf{p} ; \beta)= & e^{-\beta\left[\frac{\mathbf{p}^{2}}{2 m}+V(\mathbf{r})\right]}\left\{1+\frac{\hbar^{2} \beta^{2}}{8 m}\left[-\nabla^{2} V\right.\right. \\
& \left.\left.+\frac{\beta}{3}(\boldsymbol{\nabla V})^{2}+\frac{\beta}{3 m}(\mathbf{p} \cdot \boldsymbol{\nabla}) V\right]+0\left(\hbar^{4}\right)\right\} .
\end{aligned}
$$

The first order is just the well known Thomas-Fermi result. Higher order terms in $\hbar$ are due to the noncommutation of the kinetic and potential energy parts of the Hamiltonian:

$\mathscr{H}=-\frac{\hbar^{2}}{2 m} \nabla^{2}+V$.

These terms may be interpreted as semiclassical corrections to the Thomas-Fermi approximation. The Wigner-Kirkwood expansion (2) can be considered as an expansion in $h$ as well as in the gradients of the potential.

Integrating $C(\mathbf{r}, \mathbf{p} ; \beta)$ with respect to $\mathbf{p}$ yields the local Bloch density $C(\mathbf{r}, \beta)$. The density distribution $\rho(\mathbf{r})$ is then obtained by the inverse Laplace transform:

$\rho(\mathbf{r})=\mathscr{L}_{\beta \rightarrow \lambda}^{-1}\left[\frac{1}{\beta} C(\mathbf{r}, \beta)\right]=\frac{1}{2 \pi i} \int_{c-i \infty}^{c+i \infty} \frac{1}{\beta} e^{\lambda \beta} C(\mathbf{r}, \beta) d \beta$

where the Fermi energy $\lambda$ is determined by normalizing $\rho(\mathbf{r})$ to the given number $N$ of particles.

Laplace transforming the Wigner-Kirkwood series term by term, the extended Thomas-Fermi (ETF) expansion is obtained. It has been shown to yield an extremely rapidly converging series for the total energy of $N$ particles in various model potentials [20], although the corresponding density distributions are limited to the classically allowed region and even diverge at the classical turning points ${ }^{\star}$. Eliminating the potential and its derivatives between the ETF expressions for $\rho(\mathbf{r})$ and the kinetic energy density $\tau(\mathbf{r})$ a semiclassical functional $\tau[\rho]$ is obtained [2225] which can be used to obtain selfconsistent semiclassical results for a given effective nucleon-nucleon interaction [24-29].

Instead of Laplace inverting the Wigner-Kirkwood expansion term by term it is possible to selectively resum only those terms in (2) which contain derivatives up to a certain order of the single-particle potential V. Resumming for instance all first order derivatives of $\dot{V}$ yields after the p-integration:

$C^{(1)}(\mathbf{r}, \beta)=\left(\frac{m}{2 \pi \hbar^{2} \beta}\right)^{3 / 2} e^{-\beta V+\frac{\hbar^{2}}{24 m} \beta^{3}(\boldsymbol{V} V)^{2}}$.

This partially resummed Bloch density generates a density distribution which is well defined and continuous everywhere in space, also outside the classically allowed region [14].

In the nuclear surface, however, this density distribution, though not vanishing, is still decreasing too steeply as compared to the quantum mechanical (e.g. $\mathrm{HF}$ ) one. This is due to the important role of the curvature of the potential in this region, not taken into account in (5). Trying to go beyond the "linearized" approximation (5), Bhaduri first proposed an approximate form of the local Bloch density containing also some second order derivatives of the potential [14]:

$$
\begin{aligned}
& C^{B h}(\mathbf{r}, \beta) \\
& =\left(\frac{m}{2 \pi \hbar^{2} \beta}\right)^{3 / 2}\left[1-\frac{\hbar^{2}}{12 m} \beta^{2} \nabla^{2} V\right] e^{-\beta V+\frac{\hbar^{2}}{24 m} \beta^{3}(\boldsymbol{V} V)^{2}} .
\end{aligned}
$$

This Bloch density gives satisfactory density distributions defined everywhere in space. It also correctly reproduces the $\hbar$-expansion up to the second order of the local ETF Bloch density corresponding to (2). Resumming consistently all first and second derivatives of the potential to all powers in $\hbar$ yields the "harmonized" Bloch density. In the spherically sym-

* This divergence in coordinate space is somewhat spurious. In fact, the Wigner-transformed (ETF) density $\rho_{w}(\mathbf{r}, \mathbf{p})$ can be understood as a distribution $[21,22]$ in momentum space, from which energies and other expectation values are obtained as well defined moments 
metric case it is given by [15]:

$$
\begin{aligned}
C^{(2)}(\mathbf{r}, \beta)= & \left(\frac{m}{2 \pi \hbar^{2}}\right)^{3 / 2}\left[\frac{\hbar^{3} \omega_{r} \omega_{\perp}^{2}}{\sinh \left(\beta \hbar \omega_{r}\right) \sinh ^{2}\left(\beta \hbar \omega_{\perp}\right)}\right]^{1 / 2} \\
& \cdot e^{-\beta\left[V-\frac{V^{\prime 2}}{2 V^{\prime \prime}}\right]-\frac{m \omega_{r}}{\hbar}\left(\frac{V^{\prime}}{V^{\prime \prime}}\right)^{2} \tanh \left(\frac{\beta}{2} \hbar \omega_{r}\right)}
\end{aligned}
$$

where the derivatives $\left(V^{\prime}, V^{\prime \prime}\right)$ are taken with respect to the radial variable $r$, and where $\omega_{r}=\sqrt{\frac{V^{\prime \prime}}{m}}$ and $\omega_{\perp}=\sqrt{\frac{1}{m} \frac{V^{\prime}}{r}}$.

The expression (7) for $C(\mathbf{r}, \beta)$ is exact for a harmonic oscillator. In the general case, the approximate expressions (5) and (7) correspond to locally replacing the considered potential by a straight line or by a parabola, respectively. The expressions (6) and (7) have the advantage that the corresponding density distribution describes very well the surface region, which is not the case for the purely linearized form of (5).

\section{On the Technique of Laplace Inverting the Bloch Density}

To obtain the one-body density $\rho(\mathbf{r})$ we employ the saddle-point method for Laplace inverting $C(\mathbf{r}, \beta)$ as proposed by Bhaduri [22]. The Laplace inversion is written as:

$$
\begin{aligned}
\rho(\mathbf{r}) & =\frac{1}{2 \pi i} \int_{c-i \infty}^{c+i \infty} e^{\lambda \beta} \frac{C(\mathbf{r}, \beta)}{\beta} d \beta \\
& =\frac{1}{2 \pi i}\left(\frac{m}{2 \pi \hbar^{2}}\right)^{3 / 2} \int_{c-i \infty}^{c+i \infty} e^{\mathrm{S}(\beta)} d \beta
\end{aligned}
$$

where $c$ is a positive real number $[35,36]$.

If $\beta_{0}$ is a stationary point defined by $[\partial S / \partial \beta]_{\beta_{0}}=0$, we can expand $S(\beta)$ around $\beta_{0}$ :

$S(\beta)=S\left(\beta_{0}\right)+\sum_{n=2}^{\infty} \frac{1}{n !} \alpha_{n}\left(\beta-\beta_{0}\right)^{n}$

with

$\alpha_{n}=\left.\frac{\partial^{n} S}{\partial \beta^{n}}\right|_{\beta=\beta_{0}}$.

Furthermore, if $\beta_{0}$ is positive, we can choose the arbitrary constant $c=\beta_{0}$. Expecting that most of the contribution to the integral comes from the part of the integration contour where $S(\beta)$ is large, the expansion can be stopped after the second term and we find the usual saddle-point result:

$I=\frac{1}{i} \int_{c-i \infty}^{c+i \infty} e^{\mathrm{S}(\beta)} d \beta \simeq \frac{1}{i} e^{\mathrm{S}\left(\beta_{0}\right)} \int_{c-i \infty}^{c+i \infty} e^{\frac{\alpha_{2}}{2 !}\left(\beta-\beta_{0}\right)^{2}} d \beta$ which can be solved analytically:

$I \simeq I_{0}=e^{\mathrm{S}\left(\beta_{0}\right)} \int_{-\infty}^{\infty} e^{-\frac{\alpha_{2}}{2 !} y^{2}} d y=\sqrt{\frac{2 \pi}{\alpha_{2}}} e^{\mathrm{S}\left(\beta_{0}\right)}$

if $\alpha_{2}$ is positive and with $y=-i\left(\beta-\beta_{0}\right)$.

This may be considered as the leading term of an expansion for $I$. Taking into account the following terms in $S(\beta)$ yields:

$I=e^{S\left(\beta_{0}\right)} \int_{-\infty}^{\infty} e^{-\frac{\alpha_{2}}{2 !} y^{2}} e^{\sum_{n=3}^{\infty} \frac{i^{n} \alpha_{n}}{n !} y^{n}} d y$.

The coefficient $\alpha_{2}$ regulates the convergence of the series in the exponent of the integrand in (10). It may be used to define the reduced variable $x$ and coefficients $\tilde{\alpha}_{n}$ :

$$
x=\sqrt{\alpha_{2}} y \quad \tilde{\alpha}_{n}=\frac{\alpha_{n}}{\alpha_{2}} .
$$

Expanding the last exponential in (10) as a function of $\alpha_{2}$, and noting that the odd powers of $y$ do not contribute to the integral, we obtain:

$I=\frac{e^{S\left(\beta_{0}\right)}}{\sqrt{\alpha_{2}}} \int_{-\infty}^{\infty} e^{-\frac{x^{2}}{2}}\left[1+A_{1}(x) \frac{1}{\alpha_{2}}+A_{2}(x) \frac{1}{\alpha_{2}^{2}}+\ldots\right] d x$

Each term $A_{1}(x), A_{2}(x) \ldots$, corresponding to increasing powers of the inverse of $\alpha_{2}$ gives rise to a correction in $I$ :

$I=\sqrt{\frac{2 \pi}{\alpha_{2}}} e^{\mathrm{S}\left(\beta_{0}\right)}\left[1+C_{1}+C_{2}+\ldots\right]$

where

$C_{1}=\frac{1}{8} \frac{\alpha_{4}}{\alpha_{2}^{2}}-\frac{5}{24} \frac{\alpha_{3}^{2}}{\alpha_{2}^{3}}$

$C_{2}=-\frac{1}{48} \frac{\alpha_{6}}{\alpha_{2}^{3}}+\frac{35}{384} \frac{\alpha_{4}^{2}}{\alpha_{2}^{4}}+\frac{7}{48} \frac{\alpha_{3} \alpha_{5}}{\alpha_{2}^{4}}-\frac{35}{64} \frac{\alpha_{3}^{2} \alpha_{4}}{\alpha_{2}^{5}}+\frac{385}{1152} \frac{\alpha_{3}^{4}}{\alpha_{2}^{6}}$.

Equation (11) is exactly the result of the method of steepest descent [30] when one takes into account more than the usual leading term for the series which represents the integral $I$.

The only problem for solving (8) is thus to find $\beta_{0}$ : for that, the method of Newton is used, when the solutions of $\partial S / \partial \beta=0$ is not analytical. For the approximated Bloch densities we used (5-7), two cases may occur, in the range between $\beta=0$ and the first real positive branch-point, if any:

- one of the solutions of $\partial S / \partial \beta=0$ is real and positive, the other ones being complex conjugate in pairs. Since we are interested in semiclassical quantities, we must eliminate the complex solutions as 
possible saddle points, because they give rise to oscillating contributions. It is the same as for purely imaginary poles for the harmonized Bloch densities, which give spurious oscillations to the local density, whereas the pole at $\beta=0$ gives the semiclassical part we are interested in $[31,32]$. Thus $\beta_{0}$ is the only real positive saddle point.

- all the solutions of $\partial S / \partial \beta=0$ are real. We can in principle choose for $\beta_{0}$ any one of the positive solutions (the exact result of (8) being independent of the value of $c$ ), providing that there is no singularity of the integrand between zero and $\beta_{0}$. In the case of the Bloch density of (5) and (6) only one positive real $\beta_{0}$ solution of $\partial S / \partial \beta=0$ is found for all problems considered in this paper (harmonic oscillator and Woods-Saxon potential).

In order to illustrate the semiclassical nature of the saddle-point method, we shall consider a very simple problem where all calculations can be performed analytically. We shall be dealing with the level density of a spherical harmonic oscillator. It is the Laplace inverse of the exact partition function given in this case by:

$$
Z(\beta)=\int C(\mathbf{r}, \beta) d^{3} r=\frac{1}{4} \frac{1}{\sinh ^{3}\left(\frac{\beta}{2} \hbar \omega\right)} .
$$

The semiclassical level density is known analytically in this case. It has been shown in the ETF approach [33] to be given by:

$\tilde{g}(\varepsilon)=\frac{\varepsilon^{2}}{(\hbar \omega)^{3}}-\frac{1}{4 \hbar \omega}$

This result is rigorously the same as what is obtained with the Strutinsky averaging procedure which can be performed analytically [34].

Using the saddle-point method to evaluate the inverse Laplace transform

$g(\varepsilon)=\frac{1}{2 \pi i} \int_{c-i \infty}^{c+i \infty} e^{\varepsilon \beta} Z(\beta) d \beta$

a real saddle-point is found at $\beta_{0}=\frac{2}{\hbar \omega} \cdot \operatorname{arccoth}(x)$ where $x=\frac{2}{3} \frac{\varepsilon}{\hbar \omega}$. Taking only the leading order term of the saddle-point series (Eq. (11)) into account, one obtains

$g_{0}(\varepsilon)=\frac{1}{\sqrt{6 \pi}} \frac{1}{2 \hbar \omega}\left(x^{2}-1\right)\left(\frac{x+1}{x-1}\right)^{\frac{3}{2} x}$

which in the limit of large quantum numbers (semiclassical limit) $x \gg 1$ reduces to $g_{0}(\varepsilon)=\frac{2}{9} \frac{e^{3}}{\sqrt{6 \pi}} \frac{\varepsilon^{2}}{(\hbar \omega)^{3}} \sim 1.028 \frac{\varepsilon^{2}}{(\hbar \omega)^{3}}$

since the function

$f(x)=\left(1-\frac{1}{x^{2}}\right)\left(\frac{x+1}{x-1}\right)^{\frac{3}{2} x}$

converges for increasing $x$ very rapidly to $e^{3}$.

This result reproduces already quite nicely the leading (Thomas-Fermi) term of the semiclassical level density in (13).

If the first saddle-point correction $C_{1}$ is taken into account, one obtains similarly, in the limit $x \gg 1$ :

$g_{1}(\varepsilon) \simeq 0.9995\left[\frac{\varepsilon^{2}}{(\hbar \omega)^{3}}-\frac{36}{35} \frac{1}{4 \hbar \omega}\right]$

Including the second saddle-point correction $C_{2}$ improves the result in the sense that the coefficients of $\varepsilon^{2} /(\hbar \omega)^{3}$ and $1 / 4 \hbar \omega$ become closer to unity, in the limit $x \gg 1$, leading to

$g_{2}(\varepsilon) \simeq 0.9999\left[\frac{\varepsilon^{2}}{(\hbar \omega)^{3}}-\frac{2520}{2521} \frac{1}{4 \hbar \omega}\right]$.

In this simple case, we have shown that in the semiclassical limit of large quantum numbers, the saddlepoint method gives not only the leading order (TF) term of the average nuclear level density, but also the semiclassical corrections with their correct coefficients.

\section{Densities and Energies Obtained with Nuclear Model Potentials}

So far, we have demonstrated the validity of the saddle-point method as a mean of obtaining a semiclassical expansion for the partially resummed Bloch density. Let us now test the ability of this method to reproduce semiclassical density distributions and ground state energies using suitable model potentials. Applying simple rules for Laplace transformations [35-37] the energy can be expressed as

$$
\begin{aligned}
E=\int_{0}^{\lambda} \varepsilon g(\varepsilon) d \varepsilon & =\lambda N-\int_{0}^{\lambda} \int_{0}^{\varepsilon} g\left(\varepsilon^{\prime}\right) d \varepsilon^{\prime} d \varepsilon \\
& =\lambda N-\mathscr{L}_{\beta \rightarrow \lambda}^{-1}\left[\frac{Z(\beta)}{\beta^{2}}\right]
\end{aligned}
$$

where $g(\varepsilon)$ is the exact single particle level density and $\lambda$ is the Fermi energy determined by the normalization condition

$N=\int_{0}^{\lambda} g(\varepsilon) d \varepsilon=\mathscr{L}_{\beta \rightarrow \lambda}^{-1}\left[\frac{Z(\beta)}{\beta}\right]$ 
Table 1. Spherical harmonic oscillator.

Average energies in a spherical harmonic oscillator potential. All energies are in $\mathrm{MeV}$. The saddle-point method is used to perform the Laplace inversion (1.4) with Bloch densities (5-7), respectively in Thomas-Fermi $\left(E_{\mathrm{TF}}\right)$, linearized $\left(E_{1}\right)$, harmonized $\left(E_{2}\right)$ and Bhaduri $\left(E_{\mathrm{Bh}}\right)$ approximations. These results are reported for different numbers $N$ of particles. The last column gives the energies obtained from the exact ones by a Strutinsky smoothing procedure. The calculations have been performed with a shell spacing of $\hbar \omega=7 \mathrm{MeV}$. The constant $\hbar^{2} / 2 m$ has been taken to be $20.748 \mathrm{MeV} \mathrm{fm}^{2}[31]$

\begin{tabular}{rrrrrr}
\hline$N$ & \multicolumn{1}{c}{$E_{\text {TF }}$} & \multicolumn{1}{c}{$E_{1}$} & \multicolumn{1}{c}{$E_{2}$} & \multicolumn{1}{c}{$E_{\text {Bh }}$} & \multicolumn{1}{c}{$E_{\text {Str }}$} \\
\hline 20 & 411.1 & 397.8 & 424.8 & 424.9 & 424.6 \\
40 & 1035.8 & 1014.7 & 1057.5 & 1057.6 & 1057.2 \\
60 & 1778.6 & 1750.9 & 1807.0 & 1807.0 & 1806.5 \\
80 & 2610.1 & 2576.6 & 2644.5 & 2644.5 & 2644.0 \\
100 & 3514.6 & 3475.7 & 3554.5 & 3554.4 & 3553.8 \\
120 & 4481.8 & 4437.9 & 4526.8 & 4526.7 & 4526.1 \\
\hline
\end{tabular}

Using the exact partition function for a given problem and performing the Laplace transformations without any approximations, one obtains of course the exact quantum mechanical densities, level densities and single-particle energy sums. Semiclassical results are obtained by using either a semiclassical expansion for the partition function $Z(\beta)$, as done in the ETF method, or by applying a semiclassical technique like the saddle-point method to perform the Laplace inversions of the Bloch density like those obtained by a partial $\hbar$-resummation of the WignerKirkwood expansion.

To test the different resummation approaches, equations (5) to (7), we have calculated the average energies of different numbers $N$ of fermions in a spherical harmonic oscillator potential (without Coulomb or spin-orbit contributions). The results of these calculations are compared in Table 1 with the corresponding Strutinsky results which can be obtained analytically in this case [34]. For the sake of comparison, the results obtained in the pure Thomas-Fermi approximation have also been reported. In all these calculations, saddle-point corrections up to second order have been included.

Comparing the results obtained in the different re- summation approaches, it appears that the harmonized resummation ansatz (7) (which is the exact Bloch density in this case), and Bhaduri's approximation (6) do about equally well and reproduce the Strutinsky values to less than $1 \mathrm{MeV}$ for all systems considered, which seems very satisfactory. The linearized form (5) on the other hand misses the Strutinsky results by almost $100 \mathrm{MeV}$ in heavy systems, which again illustrates the importance of taking correctly into account the curvature of the potential. It is in fact quite striking that the results obtained in the pure Thomas-Fermi approximation are closer to the "exact" semiclassical results than those of the linearized form. Indeed, the semiclassical corrections to the Thomas-Fermi result in the Wigner-Kirkwood expansion (2) though giving relatively small contributions to the total energy, result from quite large contributions which almost cancel. It is therefore not surprising that the neglection of one of the $\hbar^{2}$-terms in the linearized approximation leads to an error of about $100 \mathrm{MeV}$ in the total energy.

The same kind of comparison has been reported in Table 2 for a spherical Woods-Saxon potential. Again the harmonized and Bhaduri's approach give good results whereas the Thomas-Fermi and the linearized approximation miss the nuclear bulk energy by $\sim 100 \mathrm{MeV}$ or more. Here again, the Thomas-Fermi approach gives better results than the linearized form.

Though quite reasonable, our results for the WoodsSaxon potential are not as close to the Strutinsky smoothed results as in the case of the harmonic oscillator, whereas the ETF approach [20] reproduces the Strutinsky energies up to $\sim 1 \mathrm{MeV}$ (which is the uncertainty of both methods). This again is related to the neglection of some terms at a given order in $h$. Of course, the fourth order terms in $h$ (including e.g. terms like $V^{4} V$ ) are not all taken into account by either of the resumation approaches, whereas they were consistently included in the ETF approach [20]. Although the total correction of or-

Table 2. Spherical Woods-Saxon potential.

Same as Table 1 for a spherical Woods-Saxon potential $\left(V_{0}=44.0 \mathrm{MeV}, a=0.67 \mathrm{fm}, R_{0}=1.27 A^{1 / 3} \mathrm{fm}\right)$ with $2 N(N=Z)$ particles, $E_{\mathrm{ETF}}$ being the extended Thomas-Fermi value [20]

\begin{tabular}{rllllll}
\hline$N=Z$ & $\begin{array}{l}E_{\mathrm{TF}} \\
(\mathrm{MeV})\end{array}$ & $\begin{array}{l}E_{1} \\
(\mathrm{MeV})\end{array}$ & $\begin{array}{l}E_{2} \\
(\mathrm{MeV})\end{array}$ & $\begin{array}{l}E_{\mathrm{Bh}} \\
(\mathrm{MeV})\end{array}$ & $\begin{array}{l}E_{\mathrm{ETF}} \\
(\mathrm{MeV})\end{array}$ & $\begin{array}{l}E_{\mathrm{StF}} \\
(\mathrm{MeV})\end{array}$ \\
\hline 20 & -717.6 & -754.0 & -686.3 & -683.5 & -680.8 & \\
36 & -1368.2 & -1420.6 & -1322.2 & -1318.2 & -1315.0 & -1315.4 \\
82 & -3344.6 & -3432.5 & -3267.1 & -3259.1 & -3254.9 & -3256.0 \\
102 & -4229.6 & -4330.6 & -4140.9 & -4131.1 & -4126.5 & -4126.6 \\
130 & -5484.5 & -5602.4 & -5381.3 & -5369.0 & -5364.0 & -5364.4 \\
146 & -6208.1 & -6335.1 & -6097.2 & -6083.5 & -6078.2 & -6078.6 \\
208 & -9044.7 & -9203.9 & -8906.3 & -8887.5 & -8881.5 & -8882.8 \\
\hline
\end{tabular}




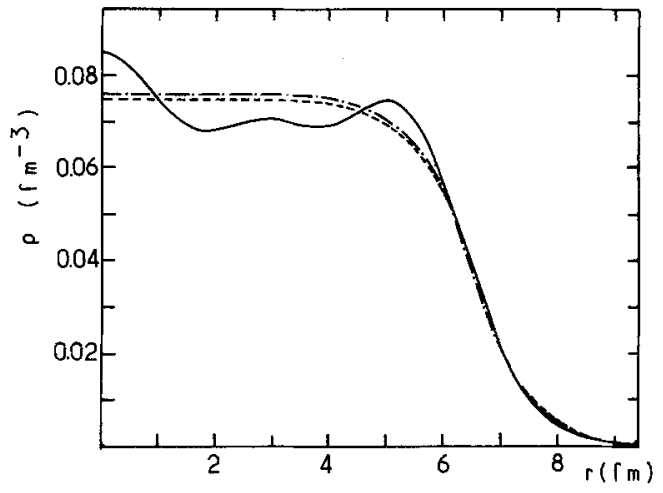

Fig. 1. Density of $N=92$ particles in a spherical Woods-Saxon potential (parameters given in Table 2). The quantum-mechanical density (full line) is compared to the semiclassical densities obtained through the saddle point method with the resummation approaches in the harmonized form (dashed-dotted line) and in Bhaduri's approximation (dashed line)

der $\hbar^{4}$ to the ETF energy is quite small (of the order of $0.1 \%$ or less), it is obtained as a sum of terms which can be quite big individually. The neglection of some of these terms in Bhaduri's approximation (6) and in the harmonized form of (7) explains the fact that the Strutinsky energies are not as well reproduced as in the ETF approach. The terms neglected in Bhaduri's form contribute apparently to a lesser extent to the total energy than those neglected by the harmonized approximation, for the problem considered here.

As mentioned in the introduction, the ETF approach fails to generate reasonable density distributions defined everywhere in space. This is not the case for the "resummed" Bloch densities which indeed correctly describe the surface properties. This is illustrated in Fig. 1 where the densities calculated by the saddle-point method with the Bloch densities of both approximations (6) and (7) are compared with the exact quantum mechanical density distribution of 92 particles in a spherical Woods-Saxon potential. Apart from quantal oscillations the latter is very well reproduced on the average.

These conclusions are not limited to one-dimensional or spherically symmetric problems. As an example, we compare in Fig. 2 contour plots of the exact and the corresponding semiclassical density distribution in an axially deformed harmonic oscillator. The exact Bloch density is:

$$
\begin{aligned}
C^{(2)}(\mathbf{r}, \beta)= & \left(\frac{m}{2 \pi \hbar^{2}}\right)^{\frac{3}{2}} \sqrt{\frac{\hbar^{3} \omega_{z} \omega_{\perp}^{2}}{\sinh \left(\beta \hbar \omega_{z}\right) \sinh ^{2}\left(\beta \hbar \omega_{\perp}\right)}} \\
& e^{-\frac{m \omega_{z}}{\hbar} z^{2} \tanh \left(\frac{\beta}{2} \hbar \omega_{z}\right)-\frac{m \omega_{\perp}}{\hbar}\left(x^{2}+y^{2}\right) \tanh \left(\frac{\beta}{2} \hbar \omega_{\perp}\right)}(1
\end{aligned}
$$

in the case of a symmetry with respect to the $z$-axis.

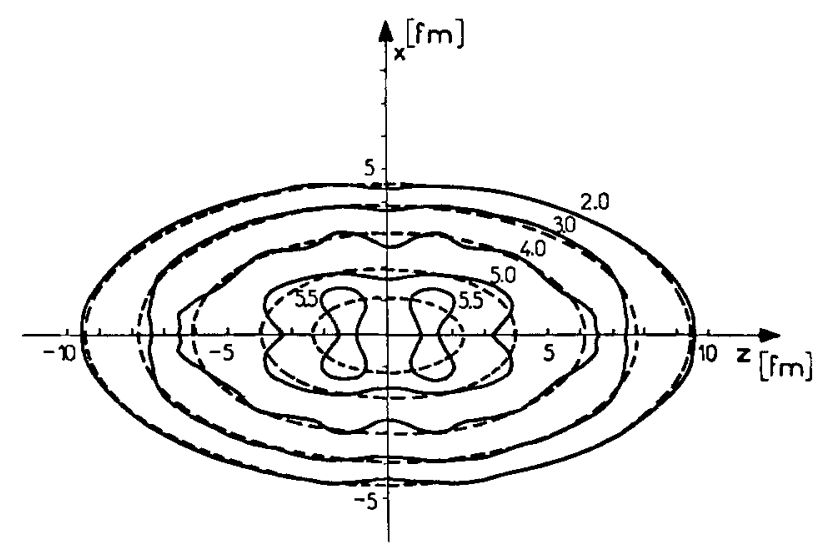

Fig. 2. Contour plot of the density distributions of 70 particles in a deformed harmonic oscillator potential of prolate shape $(15)\left(\omega_{x}\right.$ $=\omega_{y}=2 \omega_{z}$ ). The quantum-mechanical density (full line) and the semiclassical density (dashed line) are given in units of $2.10^{-2} / \mathrm{fm}^{3}$

The good reproduction of the average density distribution in the semiclassical approach is evident in this figure.

\section{Conclusions}

We have discussed how Laplace inversions of the single-particle Bloch density can be easily performed using the saddle-point method and illustrated with simple examples that the latter is indeed a semiclassical approach.

The saddle-point method, in conjunction with the resummation approximations (6) and (7) to the generally unknown exact Bloch density has been shown to provide a very powerful tool for calculating average nuclear properties for given model potentials. Not only the average energies have been reproduced to within a few $\mathrm{MeV}$ (of the order of $0,5 \%$ or less) of the corresponding Strutinsky smoothed results, but aiso the associated average density distributions are well-behaved everywhere in space. These properties have been shown to hold for different single particle model potentials and for spherical as well as for deformed shapes.

Comparing the different resummation approximations it has appeared that Bhaduri's approach is probably the best suited for a generalization to deformed shapes. It has already been extended to include Coulomb and spin-orbit interactions as well as an effective mass [13]. The method is currently generalized to excited nuclei in the statistical approximation [38].

The present method provides the foundation of an approach which may be applied in selfconsistent 
semiclassical calculations $[13,38]$. Used in connection with the Strutinsky shell-correction procedure this method is of great value for many different applications in nuclear physics where nuclear bulk properties are playing the dominant role.

The authors are indebted to Prof. R.K. Bhaduri for having initialized and largely encouraged this work. Many enlightening discussions with P. Schuck, H. Gräf, P. Quentin and M. Vallières are gratefully acknowledged.

\section{References}

1. Weizsäcker, C.F. von: Z. Phys. 96, 431 (1935)

2. Bethe, H.A., Bacher, R.F.: Rev. Mod. Phys. 8, 82 (1936)

3. Myers, W.D., Swiatecki, W.J.: Nucl. Phys. 81, 1 (1966)

4. Myers, W.D., Swiatecki, W.J.: Ann. Phys. 55, 395 (1969)

5. Myers, W.D., Swiatecki, W.J.: Ann. Phys. 84, 186 (1974)

6. Strutinsky, V.M.: Nucl. Phys. A 122, 1 (1968)

7. Strutinsky, V.M.: Nucl. Phys. A 218, 169 (1974)

8. Brack, M., Quentin, P.: Phys. Lett. 56B, 421 (1975)

9. Brack, M., Quentin, P.: Nucl. Phys. A 361, 35 (1981)

10. Vautherin, D., Brink, D.: Phys. Rev. C5, 626 (1972)

11. Beiner, M., Flocard, H., Nguyen Van Giai, Quentin, P.: Nucl. Phys. A 238, 29 (1975)

12. Bartel, J., Quentin, P., Brack, M., Guet, C., Håkansson, H.-B.: Nucl. Phys. A 386, 79 (1982)

13. Bartel, J., Vallières, M.: Phys. Lett. 114B, 303 (1982)

14. Bhaduri, R.K.: Phys. Rev. Lett. 39, 329 (1977)

15. Durand, M., Brack, M., Schuck, P.: Z. Phys. A - Atoms and Nuclei 286, 381 (1978)

16. Durand, M., Schuck, P., Brack, M.: Z. Phys. A - Atoms and Nuclei 296, 87 (1980)

17. Wigner, E.P.: Phys. Rev. 40, 749 (1932)

18. Kirkwood, J.G.: Phys. Rev. 44, 31 (1933)

19. Jennings, B.K., Bhaduri, R.K.: Nucl. Phys. A 237, 149 (1975)

20. Jennings, B.K., Bhaduri, R.K., Brack, M.: Phys. Rev. Lett. 34, 228 (1975)

21. Voros, A.: Ph. D. Thesis, Université de Paris, Orsay, 1977

22. Grammaticos, B., Voros, A.: Ann. Phys. 123, 359 (1979)

23. Grammaticos, B., Voros, A.: Ann. Phys. 129, 153 (1980)

24. Guet, C., Brack, M.: Z. Phys. A - Atoms and Nuclei 297, 247 $(1980)$

25. Bohigas, O., Campi, X., Krivine, H., Treiner, J.: Phys. Lett. 64B 381 (1976)
26. Chu, Y.H., Jennings, B.K., Brack, M.: Phys. Lett. 68B, 407 (1977)

27. Guet, C., Håkansson, H.B., Brack, M.: Phys. Lett. $97 \mathrm{~B}, 7$ (1980)

28. Brack, M.: Colloque sur les Méthodes Mathématiques de la Physique Nucléaire, Bruxelles, 21-25/6/1982. Demeur, M., Giraud, B., Quentin, P. (eds.) p. 251 (1983)

29. Brack, M., Guet, C., Håkansson, H.B.: (to be published)

30. Morse, P., Feshbach, H.: Methods of theoretical physics. Part. I. New York: Mc-Graw-Hill 1953

31. Jennings, B.K.: Ph. D. Thesis, McMaster University 1976

32. Ring, P., Schuck, P.: The nuclear many body problem. Texts and Monographs in Physics. Berlin, Heidelberg, New York: Springer-Verlag, 1980

33. Bhaduri, R.K., Ross, C.K.: Phys. Rev. Lett. 27, 606 (1971)

34. Brack, M., Pauli, H.C.: Nucl. Phys. A 207, 401 (1973)

35. Dötsch, G.: Handbuch der Laplace-Transformation. Vol. I-III, Basel: Birkhäuser Verlag 1950

36. Pol, B. Van der, Bremmer, H.: Operational calculus. Cambridge: Cambridge University Press 1955

37. Abramowitz, M., Stegun, I.A.: Handbook of mathematical functions. New York: Dover 1965

38. Bartel, J., Brack, M., Guet, C., Håkansson, H.B.: (to be published)

J. Bartel

Institut Max von Laue

Paul Langevin

B.P. 156

F-38042 Grenoble Cedex

France

and

Laboratoire de Physique Théorique

Université de Bordeaux I

Rue du solarium

F-33170 Gradignan

France

M. Durand

Institut de Sciences Nucléaires

(IN2P3)

F-38026 Grenoble Cedex

France

M. Brack

Institut für Theoretische Physik

Universität Regensburg

Universitätsstrasse 31

D-8400 Regensburg

Federal Republic of Germany 